ARTICLE

https://doi.org/10.1038/s41467-019-08878-8

\title{
Halogen bonding as a supramolecular dynamics catalyst
}

\author{
Patrick M.J. Szell ${ }^{1}{ }^{1}$, Scott Zablotny ${ }^{1} \&$ David L. Bryce ${ }^{1}{ }^{1}$
}

Dynamic processes have many implications in functional molecules, including catalysts, enzymes, host-guest complexes, and molecular machines. Here, we demonstrate via deuterium NMR relaxation experiments how halogen bonding directly impacts the dynamics in solid 2,3,5,6-tetramethylpyrazine cocrystals, catalyzing the methyl group rotation. On average, we observe a reduction of $56 \%$ in the rotational activation energy of the methyl groups in the halogen bonded cocrystals, contrasting the reduction of $36 \%$ in the hydrogen bonded cocrystals, with respect to pure 2,3,5,6-tetramethylpyrazine. Density functional theory calculations attribute this superior catalytic ability of the halogen bond to the simultaneous destabilization of the staggered conformation and stabilization of the gauche conformation, overall reducing the rotational energy barrier. Furthermore, the calculations suggest that the catalytic ability of the halogen bond may be tuneable, with stronger halogen bond donors acting as superior dynamics catalysts. Thus, halogen bonding may play a role in both assembly and promoting dynamical processes.

\footnotetext{
${ }^{1}$ Department of Chemistry and Biomolecular Sciences \& Centre for Catalysis Research and Innovation, University of Ottawa, Ottawa, ON K1N 6N5, Canada. Correspondence and requests for materials should be addressed to D.L.B. (email: dbryce@uottawa.ca)
} 
$\mathrm{T}$ he world of molecules and solids is far from static. Indeed, molecules exhibit well-known degrees of freedom, from simple rotations to more complex movements, which have been applied in the design of effective rotating catalysts ${ }^{1}$, molecular gyroscopes ${ }^{2}$, and to understand how guests, such as $\mathrm{CO}_{2}$, behave once adsorbed in a host ${ }^{3,4}$. Dynamics also play pivotal roles in much larger systems, such as biological macromolecules ${ }^{5}$, with faulty dynamical processes within enzymes being associated with disease ${ }^{6}$. Fortunately, these complex movements can be exploited, with molecular machines ${ }^{7}$ recently having risen in prominence, yielding the 2016 Nobel Prize in Chemistry ${ }^{8}$, with several configurations relying on rotations ${ }^{9-11}$, such as molecular motors $^{12,13}$ and propellers ${ }^{14}$. However, the impact of introducing interacting moieties on such dynamical processes remains unclear, and the rapid rotations of methyl groups offer an opportunity to gain insights into how molecular rotations can be affected by changes in the local chemical environment.

There have been sparse reports on how van der Waals interactions, such as those associated with inter- or intramolecular contacts, can affect the energy barrier of a methyl group's rotation $^{15-18}$. Counterintuitively, both increases and decreases in the rotational energy barrier have been observed upon the introduction of van der Waals contacts, rather than the perhaps expected consistent increase due to steric hindrance. Examples of decreases in the rotational energy barrier have been rationalized as resulting from the destabilization of the staggered conformation of the rotator, overall reducing the barrier ${ }^{15}$. This effect from van der Waals contacts on a methyl group has been observed in a select few instances in polymers ${ }^{19}$ and proteins ${ }^{20}$. In the case of proteins, increases and decreases in the methyl rotational energy barrier have both been observed, and the presence of ligands has been shown to play a critical role in their internal dynamics ${ }^{21}$. While these effects are structurally localized, they potentially could be exploited in the design of ligands and molecular machines through the incorporation of functional groups associated with a potent dynamical catalytic ability.

Halogen bonding $(\mathrm{RX} \cdots \mathrm{Y} ; \mathrm{X}=\mathrm{I}, \mathrm{Br}, \mathrm{Cl}, \mathrm{F} ; \mathrm{Y}=$ electron donor) is a non-covalent interaction akin to the hydrogen bond ${ }^{22}$ which is currently experiencing a surge of interest in supramolecular chemistry ${ }^{23,24}$, and related fields due to its directionality ${ }^{25}$ and tuneability $(\mathrm{I}>\mathrm{Br}>\mathrm{Cl} \gg \mathrm{F})^{26,27}$, allowing for the rational design of catalysts ${ }^{28,29}$, and supramolecular frameworks ${ }^{30,31}$. Halogen bonding is defined as the attraction between the region of elevated electrostatic potential ${ }^{32}$ on the halogen bond donor, associated with the electron-poor $\sigma$-hole ${ }^{33}$, and an electron-rich halogen bond acceptor, such as a Lewis base or nucleophile ${ }^{34}$. Recently, halogen bonding has been introduced in the field of supramolecular rotators, in configurations such as the molecular rotor on axle $^{35,36}$ and a molecular top ${ }^{37}$. While halogen bonding served the role of an attractive interaction to maintain a particular geometry that best favors the rotation, the direct influence of halogen bonding contacts on the molecular dynamics was not the focus of these works.

Here, we show the first instance of halogen bonding having a direct influence on local dynamical processes, with the halogen bond donor acting as a dynamics catalyst superior to the hydrogen bond. In this context, dynamics catalysis refers to the reduction of the energy barrier associated with a dynamic process, such as rotation. To this end, we employ deuterium solid-state NMR spectroscopy and spin-lattice relaxation time constant $\left(T_{1}\right)$ measurements on a series of cocrystals and salts featuring the halogen bond acceptor 2,3,5,6-tetramethylpyrazine (TMP), observing changes in the methyl group activation energy upon the introduction of a halogen bond or hydrogen bond, and comparing the results to those obtained for pure TMP and its halide salts $(\mathrm{HCl}, \mathrm{HBr})$. The TMP acceptor provides an ideal scaffold to study the effect of changes in the chemical environment on rotation, as the methyl groups are consistently located immediately adjacent to the non-covalent interaction being introduced.

\section{Results}

Structural elements. Samples 1-3, shown in Fig. 1, consist of cocrystals featuring a I $\cdots \mathrm{N}$ halogen bond [1 (1,4-diiodotetrafluorobenzene)(TMP) $38 ; 2$ (1,3,5-trifluoro-2,4,6-triiodobenzene) $(\mathrm{TMP})^{39} ; 3$ (iodine)(TMP) $]^{40}$, while samples 4 and 5 feature a $\mathrm{O}-\mathrm{H} \cdots \mathrm{N}$ hydrogen bond [4 (1,4-cyclohexanedicarboxylic acid)

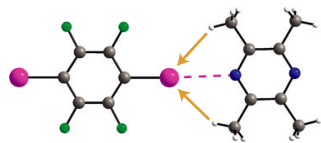<smiles>Cc1nc(C)n([I-]c2c(F)c(F)c(I)c(F)c2F)c1C</smiles>

5

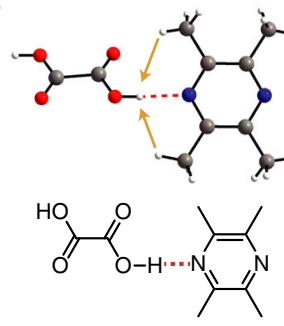

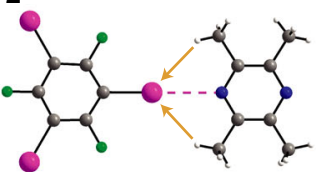

(N)

6

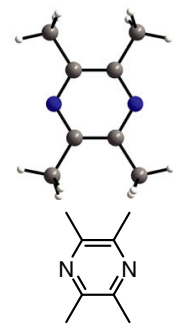

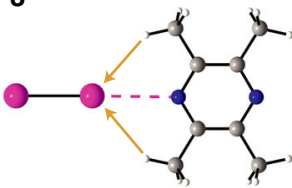

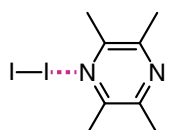

-

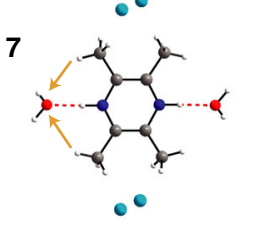

$\mathrm{Cl}$

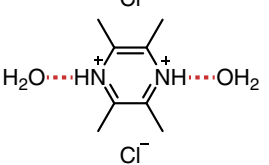

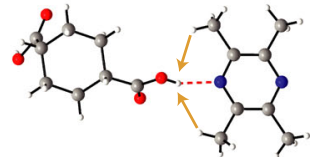

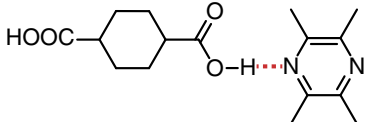

8

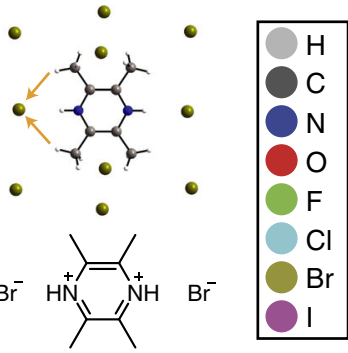

Fig. 1 Depictions of the X-ray crystal structures and molecular structures involving TMP (1-8) surveyed in this work. The dashed magenta lines denote the halogen bonds while the dashed red lines denote hydrogen bonds. The orange arrows denote close contacts between the protons on the methyl group and nearby atoms 


\section{Table 1 Methyl group rotational activation energy for each compound derived from the $T_{1}$ relaxation experiments}

\begin{tabular}{llll} 
Compound & $\mathbf{l} \cdots \mathbf{N} / \mathbf{H} \cdots \mathbf{N}$ bond length $(\mathbf{A})$ & Activation energy $\left(\mathbf{k J}\right.$ mol $\left.\mathbf{~}^{\mathbf{1}}\right)$ & Note \\
\hline 1 & $3.0665(18)$ & $2.84 \pm 0.14$ & Halogen bonded \\
2 & $2.993(3)$ & $3.24 \pm 0.13$ & Halogen bonded \\
3 & $3.075(5)$ & $3.62 \pm 0.13$ & Halogen bonded \\
4 & 1.919 & $4.17 \pm 0.09$ & Hydrogen bonded \\
5 & 1.809 & $5.02 \pm 0.06$ & Hydrogen bonded \\
6 & $\mathrm{n} / \mathrm{a}$ & $7.31 \pm 0.17$ & $\mathrm{Pure} T \mathrm{MP}$ \\
7 & $\mathrm{n} / \mathrm{a}$ & $6.81 \pm 0.16$ & $\mathrm{HCl}$ salt \\
8 & $\mathrm{n} / \mathrm{a}$ & $13.64 \pm 0.45$ & $\mathrm{HBr}$ salt \\
\hline
\end{tabular}

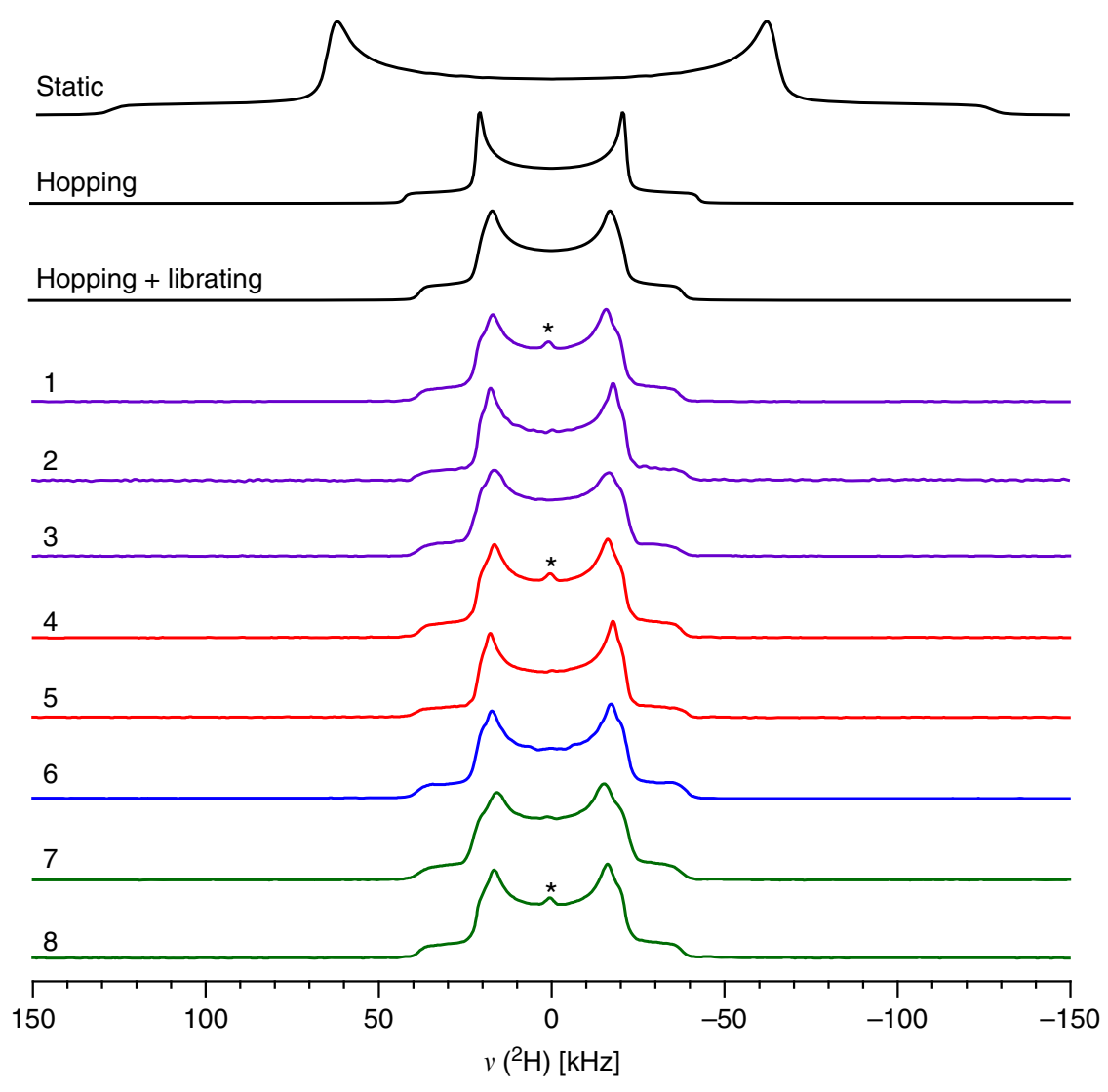

Fig. 2 Experimental static deuterium $\left({ }^{2} \mathrm{H}\right)$ solid-state NMR spectra of compounds 1-8 at room temperature. In black are the simulated spectra, in purple are the halogen bonded species, in red are the hydrogen bonded species, in blue is pure TMP, and the halide salts are in green. The asterisks denotes a trace quantity $(<1 \%)$ of $\mathrm{D}_{2} \mathrm{O}$

$(\mathrm{TMP})^{41} ; 5$ (oxalic acid)(TMP) ${ }^{41}$ ]. Sample 6 consists of pure TMP (6, TMP $)^{42}$ and serves as a reference point for the activation energy, while the halide salts [7 (2,3,5,6-tetramethylpyrazinium hydrochloride hydrate $)^{43}, \quad 8$ (2,3,5,6-tetramethylpyrazinium hydrobromide $)^{43}$ ] serve as controls for the presence of nonhalogen-bonded halogens near the methyl groups. Unfortunately, the sample quality of the hydroiodide salt ${ }^{43}$ was not satisfactory despite multiple preparatory attempts, and was therefore excluded from our analysis. The crystal structures mentioned here have been described elsewhere, with the halogen bond and hydrogen bond geometries summarized in Table 1. In brief, the halogen bond geometries are comparable across samples $\mathbf{1}, \mathbf{2}$, and $\mathbf{3}$, with $\mathbf{2}$ having the shortest halogen bond and $\mathbf{3}$ having the longest halogen bond, placing an iodine atom in proximity to the rotating methyl groups of the acceptor. The hydrogen bonding geometries are also similar in samples 4 and 5 , although 4 has a hydrogen bond $\sim 0.1 \AA$ longer than that of sample 5, with an $\mathrm{O}-\mathrm{H}$ group in proximity to the methyl groups. Compound 7 is a hydrate, with the water molecule interacting with the $\mathrm{N}-\mathrm{H}^{+}$ moiety via a $\mathrm{N}-\mathrm{H}$... O hydrogen bond. The $\mathrm{Cl}^{-}$anions in compound 7 occupy the spaces near the center between both methyl groups. In contrast, compound $\mathbf{8}$ is not a hydrate, with the $\mathrm{Br}^{-}$ anions distributed around the unit cell. Additional $\mathrm{Cl}^{-}$and $\mathrm{Br}^{-}$ anions are shown in Fig. 1 for compounds 7 and 8, respectively, to show the environment surrounding the methyl groups of interest. Across each sample, contacts to the methyl groups vary, with no clear trend in terms of distance or number of contacts. As a result, this series of compounds presents an ideal case enabling the assessment of the impact of a halogen bonding or hydrogen bonding moiety on the methyl group rotations.

Experimental activation energies. Solid-state NMR spectroscopy of deuterium, a quadrupolar nucleus $\left({ }^{2} \mathrm{H}\right.$, spin $\left.I=1\right)$, has long been used for characterizing molecular dynamics. Under static 
sample conditions, the ${ }^{2} \mathrm{H}$ solid-state NMR spectral line shape for a methyl group is diagnostic of its motion, with rapid motions typically causing an averaging effect and narrowing the spectra. Further, the quadrupolar coupling and lower magnetogyric ratio of ${ }^{2} \mathrm{H}$ renders it ideal for studies on dynamics, in contrast to ${ }^{1} \mathrm{H}$ (spin $I=1 / 2$ ) that have NMR spectra typically dominated by dipolar coupling in the solid state. With a natural abundance of $0.0115 \%$, isotopic enrichment is used to perform ${ }^{2} \mathrm{H}$ NMR experiments. This provides an opportunity to selectively label the methyl groups of interest here. Satisfactory enrichment of TMP was obtained through deuterium-proton exchange in a hot alkaline solution over one week, yielding a consistent deuterium enrichment factor across all samples.

The ${ }^{2} \mathrm{H}$ solid-state NMR spectra acquired at room temperature for each sample using a quadrupolar echo pulse sequence ${ }^{44}$ are shown in Fig. 2, with the linewidths given in Supplementary Table 2. These experiments were carried out on stationary powdered samples. The resulting spectra are superimposable, suggesting unsurprisingly that the global nature of the methyl group dynamics is unchanged across the various samples. In order to extract a full and detailed description of the dynamics, the ${ }^{2} \mathrm{H}$ line shapes were simulated using EXPRESS ${ }^{45}$ software. The spectra were fit to a threefold hopping model along with additional librational motions of the $\mathrm{CH}_{3}$ groups. This libration, also qualitatively reproduced by DFT calculations (see Supplementary Figure 5), is rationalized by the rotation of the adjacent methyl group, causing an increase in the distance between both methyl groups in the gauche position as a result of steric effects. While the rapid rotation is responsible for narrowing the line shape, the libration is manifested as a broadening of the main spectral discontinuities (see the simulations in Fig. 2 and Supplementary Figures 3 and 4). Accordingly, the narrow line shape $(35 \pm 3 \mathrm{kHz}$ horn separation) is consistent with rapidly rotating methyl groups, while there is an absence of other major dynamic processes such as a ring-flip of TMP along the halogen or hydrogen bond axis. In order to extract the thermodynamic parameters governing the methyl group rotation, the ${ }^{2} \mathrm{H}$ spinlattice $\left(T_{1}\right)$ relaxation time constants have been measured by solid-state NMR spectroscopy over a wide temperature range of $\sim 140{ }^{\circ} \mathrm{C}$ at $20^{\circ} \mathrm{C}$ intervals. These measurements were achieved by combining the quadrupolar echo ${ }^{44}$ pulse sequence with the inversion recovery experiment. The $T_{1}$ relaxation time constants were measured using the integral of the entire line shape, and were determined to be nearly isotropic by measuring the $T_{1}$ values for separate parts of the spectrum. Furthermore, throughout the temperature range, apart from sample 8 , the ${ }^{2} \mathrm{H}$ line shape remained unchanged down to $\sim 150 \mathrm{~K}$, supporting an Arrheniusdominated motion in the experimental temperature range ${ }^{46}$. A previous study on the $T_{1}$ relaxation time constants of pure TMP reveals that quantum tunneling is insignificant above temperatures of $80 \mathrm{~K}^{47}$. Consequently, in the high temperature regime, the Arrhenius activation energy can be conveniently extracted using Eq. 1: ${ }^{48}$

$$
T_{1} \propto \frac{1}{\tau_{\mathrm{c}}}=A \exp \left(\frac{-E_{\mathrm{a}}}{R T}\right)
$$

In Eq. $1, \tau_{c}$ corresponds to the correlation time, $A$ is the preexponential factor, $E_{\mathrm{a}}$ is the Arrhenius activation energy, $R$ is the gas constant, and $T$ is the temperature. The activation energy can be extracted by plotting the natural logarithm of the $T_{1}$ relaxation time constant as a function of the inverse temperature $(1 / T)$, with the slope equal to $-E_{\mathrm{a}} / R$. These data are plotted in Fig. 3 and tabulated in Supplementary Tables 3-10, with the fitting parameters given in Supplementary Table 1. Upon cooling sample

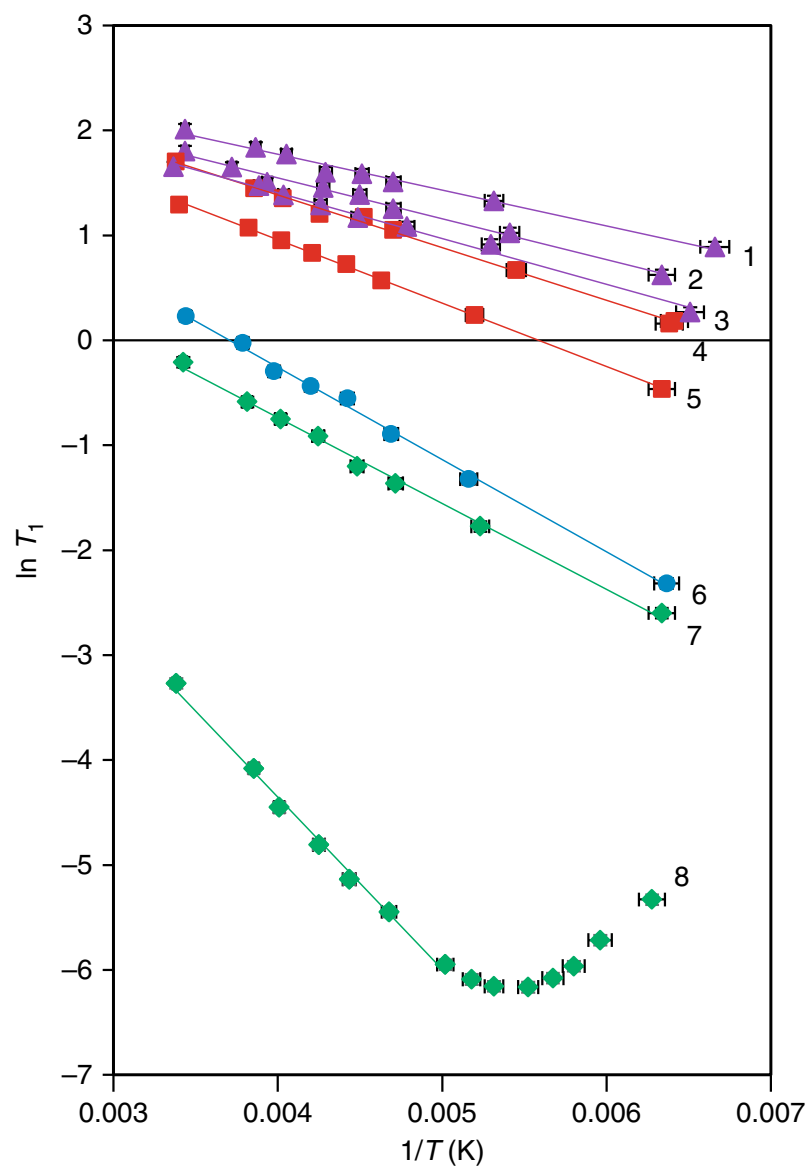

Fig. 3 Experimental In $T_{1}$ relaxation times plotted as a function of the inverse temperature $(1 / T)$. The error bars represent an upper limit on instability in the temperature and the uncertainty of the $T_{1}$ relaxation time measurements

8 to $180 \mathrm{~K}$, a line shape distortion was observed, which is associated with approaching the $T_{1}$ minimum. Consequently, supplementary data point sampling was performed for sample 8 at these lower temperatures, and the activation energy was extracted using data at higher temperatures. The activation energies are summarized in Table 1.

The activation energies associated with the methyl group rotation are lower for the cocrystals featuring a halogen bond (13 ) and a hydrogen bond (4 and 5) when compared to pure TMP (6). Moreover, the activation energies for the halogen bonded complexes were found to be significantly lower than those of the hydrogen bonded complexes, with $\mathbf{1}$ having an activation energy of nearly half that of $\mathbf{5}$, for example. Halide salt $\mathbf{7}$, which has a water molecule in proximity of the methyl groups, has a slightly lower activation energy when compared to the pure acceptor, while 8 has the highest activation energy, at $186 \%$ more than that of pure TMP.

Computational chemistry. To support the experimental results, a series of DFT calculations was carried out using the Amsterdam Density Functional (ADF) ${ }^{49-51}$ software package on the cocrystals featuring perfluorinated halogen bond donors, in order to provide a theoretical basis for the observed reductions in the activation energy. The models of $\mathbf{1}$ and $\mathbf{2}$ consisted of TMP interacting with one nearby halogen bond donor, identical to the crystal structure depictions shown in Fig. 1. A series of linear transit calculations have been performed, altering the $\mathrm{H}-\mathrm{C}-\mathrm{C}-\mathrm{C}$ dihedral angle from $-180^{\circ}$ to $90^{\circ}$, in steps of $2.5^{\circ}$, for the methyl 


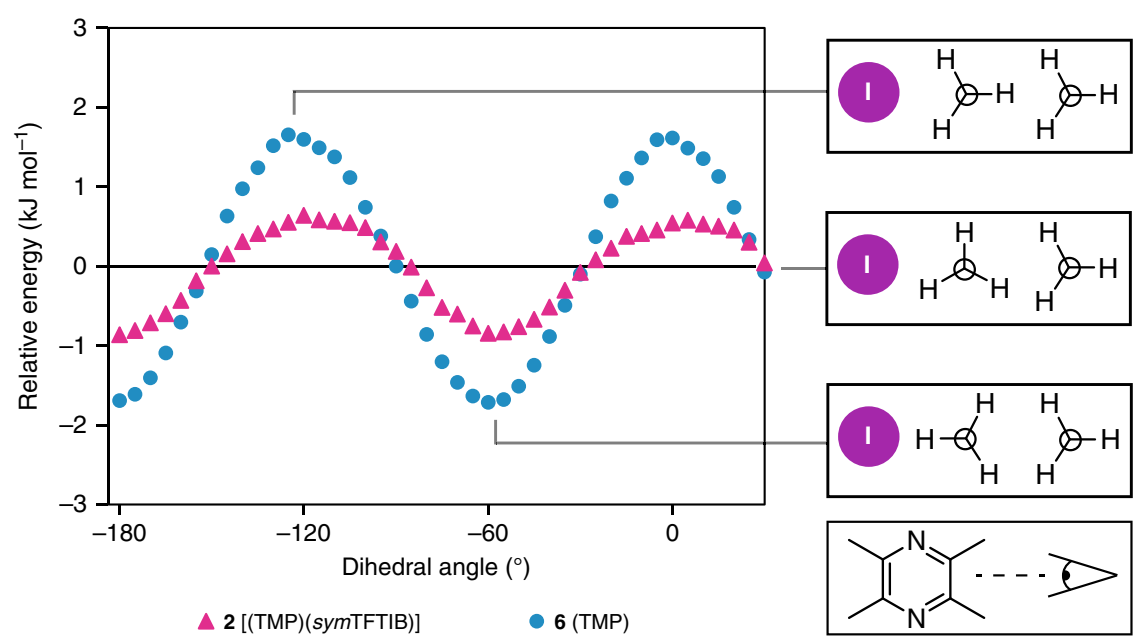

Fig. 4 DFT-calculated relative energy as a function of the methyl group dihedral angle (H-C-C-C). The blue circles denote pure TMP (6) and the magenta triangles denote cocrystal $\mathbf{2}$. A diagram showing the methyl group geometry at the energy maximum, zero-crossing, and minimum is shown on the right. A depiction showing the perspective in the diagram is shown on the bottom right

group closest to the halogen bond donor. The energy was calculated at every point of the methyl group rotation after a geometry optimization cycle, while maintaining a constrained dihedral angle. The calculated activation energies were approximated by subtracting the energy minimum from the energy maximum. For both models, the energy minimum of a single methyl group rotation consisted of the staggered conformation (methyl groups $180^{\circ}$ out of phase), while the energy maximum consisted of the gauche conformation (methyl groups in-phase). As a result of only rotating a single methyl group, the energy associated with that rotation could be estimated.

The results of the DFT calculations are shown in Fig. 4, comparing the relative energy of compounds $\mathbf{2}$ and $\mathbf{6}$, upon the rotation of the methyl group. The results comparing structure $\mathbf{1}$ to structure $\mathbf{6}$ are shown in Supplementary Figure 6. The pseudosinusoidal nature of the energy upon the rotation of the methyl group is consistent with a $C_{3}$ rotation, with three energy minima in a full $360^{\circ}$ rotation due to the presence of three hydrogen atoms on the methyl group. Upon the introduction of a halogen bond, there is a clear increase in the energy minimum and a decrease in the energy maximum, with an overall energy barrier reduction of $58 \%$ for 1 and $56 \%$ for 2 when compared to pure TMP, in agreement with the experimental values. The orientation of the adjacent methyl group not interacting with iodine did not significantly impact the overall catalytic effect, with the results of the calculations shown in Supplementary Figure 7.

A series of calculations was then carried out comparing the effect of the halogen bond length $\left(d_{\mathrm{I} \ldots \mathrm{N}}\right)$ on the methyl group activation energy, shown in Fig. 5. This was performed by calculating the energy difference between the in-phase and outof-phase conformations on a model of TMP interacting with either iodomethane or iodotrifluoromethane, varying the distance between both fragments from an $R_{\mathrm{XB}}$ value of 1.10 to 0.75 , at 0.05 increments. For clarity, here we use $R_{\mathrm{XB}}$ to refer to the quotient of the halogen bond length $\left(d_{\mathrm{I} \ldots \mathrm{N}}\right)$ and the sum of their van der Waals radii $\left(R_{\mathrm{XB}}=d_{\mathrm{I} \ldots \mathrm{N}} / \sum d_{\mathrm{vdw}}\right)$.

As shown in Fig. 5, the I...N distance plays a clear role in reducing the rotational activation energy barrier, with the catalytic abilities of the halogen bond being most pronounced between $R_{\mathrm{XB}}$ of 0.85 and 0.95 . The difference in the energy curves between iodomethane and iodotrifluoromethane presented in Fig. 5 is quite substantial, suggesting that the catalytic effect associated with the halogen bond isn't merely due to the presence of an iodine atom, but the choice of the donor moiety may also

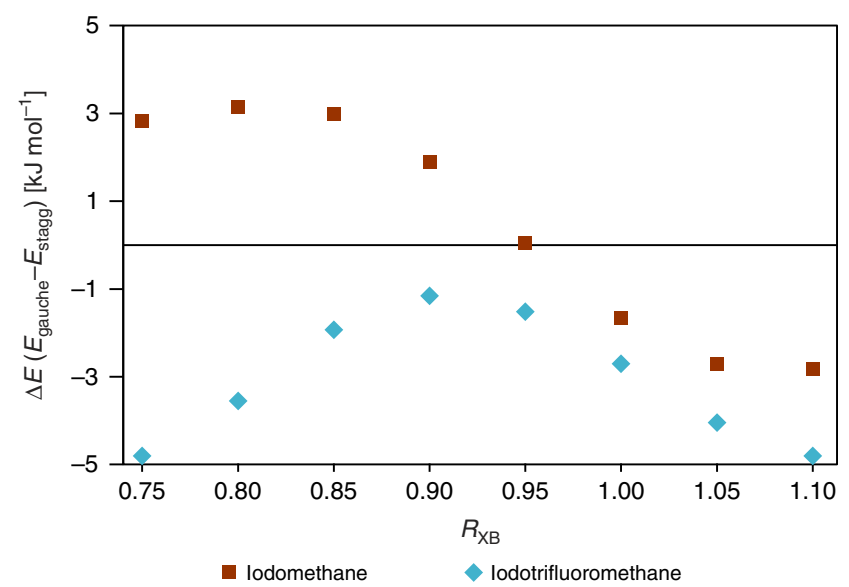

Fig. 5 Calculated energy difference between the staggered and gauche conformations of TMP as a function of the $1 \cdots \mathrm{N}$ halogen bond length (expressed as $R_{X \mathrm{~B}}$ ), interacting with either iodomethane (red squares) or iodotrifluoromethane (blue diamonds)

play a role. In the case of the non-fluorinated iodomethane, the energy barrier crosses the zero energy point at $R_{\mathrm{XB}}=0.95$, suggesting that the gauche conformation becomes energetically favorable rather than the staggered conformation at short I $\cdots \mathrm{N}$ distance. In contrast, when interacting with iodotrifluoromethane, the staggered conformation remains energetically favorable as the halogen bond distance is decreased, reaching an energy minimum at an $R_{\mathrm{XB}}$ of 0.90 . We refer to this effect as catalytic because the halogen bond is not consumed or destroyed during the process of methyl rotation.

\section{Discussion}

The experimental activation energies, summarized in Table 1 and Fig. 6, are lowest for the halogen bonded cocrystals (1-3), followed by the hydrogen bonded cocrystals $(\mathbf{4}, \mathbf{5})$, the pure TMP (6) and the halide salts $(7,8)$. Furthermore, the activation energies of 1-3 are amongst the lowest in the literature for a methyl group rotation, comparable to 2,6-dimethylnaphthalene and 1,3,5-trichloro-2,4,6-trimethylbenzene, having activation energy of $1.6^{18}$ and $2.4 \mathrm{~kJ} \mathrm{~mol}^{-152}$, respectively. In the case of the latter, the low energy barrier has been attributed to the chlorine 

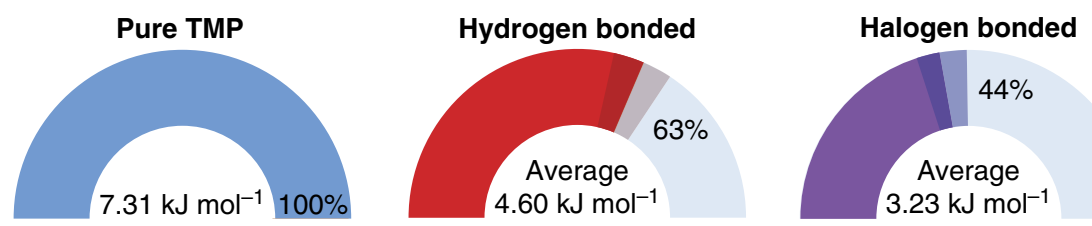

Fig. 6 Summary of the reduction in the experimentally measured activation energy associated with the rotation of the methyl groups. The average activation energies are given for the halogen bonded and hydrogen bonded cocrystals

substitution, in contrast to the $8.0 \mathrm{~kJ} \mathrm{~mol}^{-1}$ barrier observed in hexamethylbenzene $e^{53}$. As a point of reference, the rotational activation energy of a methyl group in organic molecules typically spans from 6.3 to $24 \mathrm{~kJ} \mathrm{~mol}^{-1} 47,54$. The reduction in the methyl group activation energy observed in the cocrystals studied here is attributed to the presence of the halogen bond donor and hydrogen bond donor in proximity to the methyl groups, as no clear trends can be discerned relating the number of contacts to the methyl groups (see Supplementary Figures $1 \& 2$ ) or to the crystal packing. Furthermore, the mere presence of halogen atoms in proximity to the methyl groups was not sufficient to catalyze the methyl group rotation, with compound $\mathbf{8}$ instead exhibiting a significant increase in the activation energy. The slight decrease in the activation energy observed in compound 7 can be rationalized by the presence of a water molecule participating in a $\mathrm{O} \cdots \mathrm{N}$ contact near the methyl groups, despite the presence of chloride anions. As a result, the catalytic effect observed in compounds 1-3 appears to be unique to covalently bonded halogens.

The DFT results on isolated models of 1, 2, and $\mathbf{6}$ (Fig. 4 and Supplementary Figure 6) suggest that this catalytic effect associated with the halogen bond is a result of the destabilization of the staggered conformation and the stabilization of the gauche conformation. Consequently, the difference between the energy maximum and minimum is reduced in the halogen bonded cocrystals, causing a net reduction in the activation energy. This is in contrast to the previous theoretical investigations on the catalytic effect of van der Waals contacts involving hydrogen on methyl groups, revealing primarily an increase in the energy minimum ${ }^{15}$. A potential mechanism for the destabilization of the staggered conformation may be due to the repulsion between the protons of the methyl group, which carry a positive electrostatic potential $\left(+74.5 \mathrm{~kJ} \mathrm{~mol}^{-1}\right.$; see Supplementary Figure 9), and the positive $\sigma$-hole of the halogen bond donor. As the electrostatic potential between the protons of the methyl group is slightly negative $\left(-5.7 \mathrm{~kJ} \mathrm{~mol}^{-1}\right)$, this may cause an attractive interaction to the $\sigma$-hole of the halogen bond, leading to a stabilizing effect. In this respect, a larger $\sigma$-hole would lead towards a stronger hydrogen...iodine interaction, further reducing the rotational energy barrier. (Another less plausible mechanism for the reduction of the energy barrier could be related to the skeletal flexing of TMP upon halogen bonding. However, an inspection of the crystal structures shows that the structural changes induced upon halogen bonding are tiny (e.g., $\mathrm{C}-\mathrm{C}-\mathrm{CH}_{3}$ angle in TMP changes by $1^{\circ}$ and distance between the two methyl carbons changes by $\sim 0.01 \AA$ ). It is not clear how these changes would result in a lower activation energy in the halogen bonded systems.)

The DFT results suggest that the distance between the iodine and the methyl rotors plays an important role in determining the catalytic ability of the halogen bond, being most significant for $R_{\mathrm{XB}}$ values between 0.85 and 0.95 . With an average $R_{\mathrm{XB}}$ value for the C-I...N fragment of 0.85 according to the Cambridge Structural Database ${ }^{55}$, these halogen bonds seem to be in the ideal range to act as a dynamical catalyst. When comparing the catalytic ability of iodomethane and iodotrifluoromethane as a function of the I...N distance by DFT calculations, a striking difference between both curves is observed, suggesting that the addition of electron withdrawing groups may alter the catalytic abilities of the halogen atom, and may allow for a tuneable dynamics catalyst. This is supported experimentally, with the catalytic ability of the halogen bond donor following the calculated molecular electrostatic potential of the $\sigma$-hole (see Supplementary Figure 8). The calculated potential of the $\sigma$-hole, highest in 1 at $168 \mathrm{~kJ} \mathrm{~mol}^{-1}$, followed by 2 at $162 \mathrm{~kJ} \mathrm{~mol}^{-1}$, and 3 at $155 \mathrm{~kJ} \mathrm{~mol}^{-1}$, satisfyingly follows the order of reduction in the methyl group rotational energy barrier.

In conclusion, we have shown how halogen bonding plays a direct role as a supramolecular dynamics catalyst through the use of ${ }^{2} \mathrm{H}$ solid-state NMR relaxation experiments. In the cases shown here, the catalytic impact of the halogen bond surpasses that of the hydrogen bond. Computational support suggests that this is achieved by destabilizing the staggered conformation and stabilizing the gauche conformation, resulting in an overall reduction of the activation energy barrier. Further, this catalytic effect may be tuneable by changing the electron withdrawing substitutions adjacent to the halogen bond donor. Consequently, the halogen bond may not only be a versatile interaction useful in crystal engineering, but may bring a unique ability of promoting dynamics within functional molecules, such as enzymes, catalysts, and molecular machines.

\section{Methods}

Sample preparation. 2,3,5,6-tetramethylpyrazine (98\%), 1,4-diiodotetrafluorobenzene (99\%), iodine (99\%), oxalic acid (99\%), and hydrobromic acid (48\%) were purchased from Sigma Aldrich. 1,3,5-trifluoro-2,4,6-triiodobenzene was purchased from Alfa Aesar. 1,4-cyclohexanedicarboxylic acid (99\%) was purchased from Acros Organics. Deuterium oxide (>99\% deuterium) was purchased from Cambridge Isotope Laboratories. Hydrochloric acid ( 38\%) and all solvents were purchased from Fisher Scientific.

2,3,5,6-tetramethylpyrazine was deuterated following a modified literature procedure $^{56}$. To a $50 \mathrm{~mL}$ glass pressure tube, $35 \mathrm{~mL}$ of a $0.6 \mathrm{M} \mathrm{NaOH}$ solution in $\mathrm{D}_{2} \mathrm{O}$ was added, followed by the addition of $2.05 \mathrm{~g}$ of 2,3,5,6-tetramethylpyrazine. The glass vessel was placed in the oven at $90^{\circ} \mathrm{C}$, at which point the $2,3,5,6-$ tetramethylpyrazine dissolved completely, and left for 7 days. The glass pressure tube was removed from the oven and allowed to return to room temperature, allowing for the deuterated 2,3,5,6-tetramethylpyrazine to crystallize. The solution was filtered and rinsed with water. The product was sublimed (yield $=57 \%$ ), affording 2,3,5,6-tetramethylpyrazine with a deuterium enrichment factor of $\sim 60.1 \%$. Compounds $\mathbf{1}^{38}, \mathbf{2}^{39}, \mathbf{7}^{43}$, and $\mathbf{8}^{43}$ were reproduced using their literature procedure using the deuterated product. Compound $3^{40}$ was prepared by cosublimation ${ }^{57}$ by combining $\mathrm{I}_{2}$ and the deuterated 2,3,5,6-tetramethylpyrazine in a round bottom sublimation apparatus. Compounds $4^{41}$ and $5^{41}$ were prepared mechanochemically using a Retsch MM400 ball mill, by placing both the deuterated 2,3,5,6-tetramethylpyrazine and hydrogen bond donor at equimolar quantities in a $10 \mathrm{~mL}$ stainless steel ball mill jar. The products were ground for $30 \mathrm{~min}$ at a frequency of $25 \mathrm{~Hz}$, using two stainless steel milling balls. The crystal structure for each powdered sample was verified by powder X-ray diffraction on a Rigaku Ultima IV instrument unless otherwise stated, with $2 \theta$ ranging from 5 to $65^{\circ}$ in increments of $0.02^{\circ}$ using $\mathrm{Cu} \mathrm{Ka}$ radiation. The powder X-ray diffractograms are shown in Supplementary Figures 10 to 17.

${ }^{2} \mathbf{H}$ solid-state NMR. All ${ }^{2} \mathrm{H}$ solid-state NMR experiments were performed at 4.7T $\left(v_{\mathrm{L}}\left({ }^{2} \mathrm{H}\right)=30.7 \mathrm{MHz}\right)$ using a Bruker Avance III console and a single-channel wideline probe fitted with a $5 \mathrm{~mm}$ coil. Static ${ }^{2} \mathrm{H}$ spectra were acquired using a quadrupolar echo ${ }^{44}(\pi / 2-\tau-\pi / 2-\tau)$ pulse sequence, using a $4.7 \mu \mathrm{s} \pi / 2$ pulse length, and a $20 \mu \mathrm{s} \tau$ delay. The $T_{1}$ relaxation experiments were performed using a quadrupolar echo coupled with an inversion recovery sequence at variable temperatures. The temperature was controlled by flowing dry nitrogen gas through 
a heat exchanger submerged in liquid nitrogen, and subsequently heated inside the probe to the desired temperature. The temperature was regulated using a Bruker variable temperature controller with the thermocouple placed near the sample, while the sample temperature was measured by placing a copper/constantan thermocouple directly on the RF coil, while using an ice water bath as a reference.

Computational. The DFT calculations were performed using the Amsterdam Density Functional software (ADF 2017) ${ }^{49-51}$. Models were built from the experimental atomic coordinates taken from the crystal structures, featuring one halogen bond donor interacting with 2,3,5,6-tetramethylpyrazine. Each model was initially optimized using the TZ2P basis set, accounting for dispersion forces using Grimme3 BJDAMP ${ }^{58}$ and relativistic effects using ZORA $^{59}$. Linear transit calculations were performed using the TZ2P basis set by rotating the methyl group at steps of $2.5^{\circ}$ relative to the $\mathrm{H}-\mathrm{C}-\mathrm{C}-\mathrm{C}$ dihedral angle, performing a geometry optimization routine at each step, and calculating the energy. Single point energy calculations were performed using pre-optimized models of the staggered and gauche conformers with variable distances and halogen bond donor fragments. The QZ4P basis set was used for all iodine atoms in the linear transit and single point energy calculations. The molecular electrostatic surface potential surfaces were generated using Gaussian $09^{60}$ with the B3LYP functional and the 3-21G basis set, and visualized using GaussView ${ }^{61}$ at 0.002 e a.u. ${ }^{-1}$. The resulting molecular electrostatic potential values for the $\sigma$-hole were found to be in agreement with previous reports ${ }^{62}$.

\section{Data availability}

The authors declare that all data supporting the findings of this study are available within the paper and its supplementary information files.

Received: 17 October 2018 Accepted: 4 February 2019

Published online: 22 February 2019

\section{References}

1. Biswas, P. K., Saha, S., Paululat, T. \& Schmittel, M. Rotating catalysts are superior: suppressing product inhibition by anchimeric assistance in fourcomponent catalytic machinery. J. Am. Chem. Soc. 140, 9038-9041 (2018).

2. Godinez, C. E., Zepeda, G. \& Garcia-Garibay, M. A. Molecular compasses and gyroscopes. II. Synthesis and characterization of molecular rotors with axially substituted bis[2-(9-triptycyl)ethynyl]arenes. J. Am. Chem. Soc. 124 4701-4707 (2002).

3. Wang, W. D., Lucier, B. E. G., Terskikh, V. V., Wang, W. \& Huang, Y. Wobbling and hopping: studying dynamics of $\mathrm{CO}_{2}$ adsorbed in metal-organic frameworks via ${ }^{17} \mathrm{O}$ solid-state NMR. J. Phys. Chem. Lett. 5, 3360-3365 (2014).

4. Zhang, Y., Lucier, B. E. G. \& Huang, Y. Deducing $\mathrm{CO}_{2}$ motion, adsorption locations and binding strengths in a flexible metal-organic framework without open metal sites. Phys. Chem. Chem. Phys. 18, 8327-8341 (2016).

5. Davey, J. A., Damry, A. M., Goto, N. K. \& Chica, R. A. Rational design of proteins that exchange on functional timescales. Nat. Chem. Biol. 13, 1280-1285 (2017).

6. Schuetz, A. K. \& Kay, L. E. A dynamic molecular basis for malfunction in disease mutants of p97/VCP. eLife 5, e20143 (2016).

7. Erbas-Cakmak, S., Leigh, D. A., McTernan, C. T. \& Nussbaumer, A. L. Artificial molecular machines. Chem. Rev. 115, 10081-10206 (2015).

8. The Nobel Prize in Chemistry 2016. https://www.nobelprize.org/nobel_prizes/ chemistry/laureates/2016/ (2016).

9. Kelly, T. R., De Silva, H. \& Silva, R. A. Unidirectional rotary motion in a molecular system. Nature 401, 150-152 (1999).

10. Karlen, S. D. et al. Symmetry and dynamics of molecular rotors in amphidynamic molecular crystals. Proc. Natl. Acad. Sci. USA. 107, 14973-14977 (2010).

11. Jiang, X., Rodríguez-Molina, B., Nazarian, N. \& Garcia-Garibay, M. A. Rotation of a bulky triptycene in the solid state: toward engineered nanoscale artificial molecular machines. J. Am. Chem. Soc. 136, 8871-8874 (2014).

12. $\mathrm{Wu}, \mathrm{Y}$. et al. A multistage rotational speed changing molecular rotor regulated by $\mathrm{pH}$ and metal cations. Nat. Commun. 9, 1953 (2018).

13. Koumura, N., Zijlstra, R. W. J., van Delden, R. A., Harada, N. \& Feringa, B. L. Light-driven monodirectional molecular rotor. Nature 401, 152-155 (1999).

14. Simpson, C. D. et al. Nanosized molecular propellers by cyclodehydrogenation of polyphenylene dendrimers. J. Am. Chem. Soc. 126, 3139-3147 (2004).

15. Baudry, J. van der Waals interactions and decrease of the rotational barrier of methyl-sized rotators: a theoretical study. J. Am. Chem. Soc. 128, 11088-11093 (2006).
16. Hembree, W. I. \& Baudry, J. Three-dimensional mapping of microenvironmental control of methyl rotational barriers. J. Phys. Chem. B 115, 8575-8580 (2011).

17. Khazaei, S. \& Sebastiani, D. Methyl rotor quantum states and the effect of chemical environment in organic crystals: $\gamma$-picoline and toluene. J. Chem. Phys. 145, 234506 (2016).

18. Wang, X. et al. Intramolecular and intermolecular contributions to the barriers for rotation of methyl groups in crystalline solids: electronic structure calculations and solid-state NMR relaxation measurements. J. Org. Chem. 76, 5170-5176 (2011)

19. Alvarez, F., Alegría, A., Colmenero, J., Nicholson, T. M. \& Davies, G. R. Origin of the distribution of potential barriers for methyl group dynamics in glassy polymers: a molecular dynamics simulation in polyisoprene. Macromolecules 33, 8077-8084 (2000).

20. Baudry, J. \& Smith, J. C. Can proteins and crystals self-catalyze methyl rotations? J. Phys. Chem. B 109, 20572-20578 (2005).

21. Krishnan, M. \& Smith, J. C. Response of small-scale, methyl rotors to proteinligand association: a simulation analysis of calmodulin-peptide binding. J. Am. Chem. Soc. 131, 10083-10091 (2009).

22. Metrangolo, P., Neukirch, H., Pilati, T. \& Resnati, G. Halogen bonding based recognition processes: a world parallel to hydrogen bonding. Acc. Chem. Res. 38, 386-395 (2005).

23. Gilday, L. C. et al. Halogen bonding in supramolecular chemistry. Chem. Rev 115, 7118-7195 (2015).

24. Cavallo, G. et al. The halogen bond. Chem. Rev. 116, 2478-2601 (2016)

25. Huber, S. M., Scanlon, J. D., Jimenez-Izal, E., Ugalde, J. M. \& Infante, I. On the directionality of halogen bonding. Phys. Chem. Chem. Phys. 15, 10350-10357 (2013).

26. Riley, K. E. et al. Halogen bond tunability I: the effects of aromatic fluorine substitution on the strengths of halogen-bonding interactions involving chlorine, bromine, and iodine. J. Mol. Model. 17, 3309-3318 (2011).

27. Riley, K. E. et al. Halogen bonding tunability II: the varying roles of electrostatic and dispersion contributions to attraction in halogen bonds. J. Mol. Model. 19, 4651-4659 (2013).

28. Chan, Y.-C. \& Yeung, Y.-Y. Halogen bond catalyzed bromocarbocyclization. Angew. Chem. Int. Ed. 57, 3483-3487 (2018).

29. Carreras, L., Serrano-Torné, M., van Leeuwen, P. W. N. M. \& Vidal-Ferran, A XBphos-Rh: a halogen-bond assembled supramolecular catalyst. Chem. Sci. 9, 3644-3648 (2018)

30. Lieffrig, J., Jeannin, O. \& Fourmigué, M. Expanded halogen-bonded anion organic networks with star-shaped iodoethynyl-substituted molecules: from corrugated $2 \mathrm{D}$ hexagonal lattices to pyrite-type 2 -fold interpenetrated cubic lattices. J. Am. Chem. Soc. 135, 6200-6210 (2013).

31. Kumar, V. et al. Halogen bonded Borromean networks by design: topology invariance and metric tuning in a library of multi-component systems. Chem. Sci. 8, 1801-1810 (2017).

32. Politzer, P. \& Murray, J. S. $\sigma$-hole interactions: perspectives and misconceptions. Crystals 7, 212 (2017).

33. Clark, T., Hennemann, M., Murray, J. S. \& Politzer, P. Halogen bonding: the б-hole. J. Mol. Model. 13, 291-296 (2007).

34. Desiraju, G. R. et al. Definition of the halogen bond (IUPAC recommendations 2013). Pure Appl. Chem. 85, 1711-1713 (2013).

35. Catalano, L. et al. Dynamic characterization of crystalline supramolecular rotors assembled through halogen bonding. J. Am. Chem. Soc. 137, 15386-15389 (2015)

36. Catalano, L. et al. Rotational dynamics of diazabicyclo[2.2.2]octane in isomorphous halogen-bonded co-crystals: entropic and enthalpic effects. J. Am. Chem. Soc. 139, 843-848 (2017).

37. Lemouchi, C. et al. Ultra-fast rotors for molecular machines and functional materials via halogen bonding: crystals of 1,4-bis(iodoethynyl)bicyclo[2.2.2] octane with distinct gigahertz rotation at two sites. J. Am. Chem. Soc. 133 6371-6379 (2011).

38. Syssa-Magalé, J.-L., Boubekeur, K., Palvadeau, P., Meerschaut, A. \& Schöllhorn, B. The tailoring of crystal structures via the self-assembly of organic coordination compounds by N...I non-covalent halogen bonds: cocrystal of sterically hindered N-heterocycles and 1,4-diiodotetrafluorobenzene. CrystEngComm 7, 302-308 (2005).

39. Szell, P. M. J. et al. ${ }^{13} \mathrm{C}$ and ${ }^{19} \mathrm{~F}$ solid-state NMR and X-ray crystallographic study of halogen-bonded frameworks featuring nitrogen-containing heterocycles. Acta Cryst. C73, 157-167 (2017).

40. Bailey, R. D., Buchanan, M. L. \& Pennington, W. T. Molecular complexes of 1,4-diazines with iodine. Acta Cryst. C48, 2259-2262 (1992).

41. Weyna, D. R., Shattock, T., Vishweshwar, P. \& Zaworotko, M. J. Synthesis and structural characterization of cocrystals and pharmaceutical cocrystals: mechanochemistry vs slow evaporation from solution. Cryst. Growth Des. 9, 1106-1123 (2009).

42. Braam, A. W. M., Eshuis, A. \& Vos, A. Tetramethylpyrazine at 300 and $100 \mathrm{~K}$. Acta Cryst. B37, 730-732 (1981). 
43. Lu, J. \& Kochi, J. K. Charge-modulated associates of anionic donors with cationic $\pi$-acceptors: crystal structures of ternary synthons leading to molecular wires. Cryst. Growth Des. 9, 291-296 (2009).

44. Davis, J. H., Jeffrey, K. R., Bloom, M., Valic, M. I. \& Higgs, T. P. Quadrupolar echo deuteron magnetic resonance spectroscopy in ordered hydrocarbon chains. Chem. Phys. Lett. 42, 390-394 (1976).

45. Vold, R. L. \& Hoatson, G. L. Effects of jump dynamics on solid state nuclear magnetic resonance line shapes and spin relaxation times. J. Magn. Reson. 198, 57-72 (2009).

46. Kowalewski, J. \& Liljefors, T. On the relationship between the potential barrier and the activation energy for the internal rotation of a methyl group. Chem. Phys. Lett. 64, 170-174 (1979).

47. Takeda, S. \& Chihara, H. Methyl rotation in 1,2,4,5-tetramethylbenzene and tetramethylpyrazine in the solid state. Tunneling spectra by proton NMR. J. Magn. Reson. 54, 285-299 (1983).

48. Spěváček, J. \& Schneider, B. Determination of the activation energies of relaxation processes from integrated intensities of the NMR bands of high resolution spectra. Czech. J. Phys. B24, 593-604 (1974).

49. Te Velde, G. et al. Chemistry with ADF. J. Comp. Chem. 22, 931-967 (2001).

50. Fonseca Guerra, C., Snijders, J. G., te Velde, G. \& Baerends, E. J. Towards an order-N DFT method. Theo. Chem. Acc. 99, 391-403 (1998).

51. Baerends, E. J. et al. ADF2017, SCM, Theoretical Chemistry, Vrije Universiteit, Amsterdam, The Netherlands. http://www.scm.com (2014).

52. Takeda, S., Fujiwara, T. \& Chihara, H. Methyl rotation in 1,3,5-trichloro-2,4,6trimethylbenzene as studied by nuclear magnetic resonance: a comparison with methyl rotation in hexamethylbenzene. J. Phys. Soc. Jpn. 58, 1793-1800 (1989).

53. Allen, P. S. \& Cowking, A. Nuclear magnetic resonance study of molecular motions in hexamethylbenzene. J. Chem. Phys. 47, 4286-4289 (1967)

54. Beckmann, P. A. Nonexponential ${ }^{1} \mathrm{H}$ spin-lattice relaxation and methyl group rotation in molecular solids. Solid State Nucl. Magn. Reson. 71, 91-95 (2015).

55. Groom, C. R., Bruno, I. J., Lightfoot, M. P. \& Ward, S. C. The Cambridge Structural Database. Acta Cryst. B72, 171-179 (2016).

56. Vincent, J. S. Paramagnetic resonance of the triplet state of tetramethylpyrazine. J. Chem. Phys. 47, 1830-1833 (1967).

57. Szell, P. M. J. et al. Cosublimation: a rapid route toward otherwise inaccessible halogen-bonded architectures. Cryst. Growth Des. 18, 6227-6238 (2018).

58. Grimme, S., Ehrlich, S. \& Goerigk, L. Effect of the damping function in dispersion corrected density functional theory. J. Comput. Chem. 32, 1456-1465 (2011).

59. van Lenthe, E., Ahlers, A. \& Baerends, E.-J. Geometry optimizations in the zero order regular approximation for relativistic effects. J. Chem. Phys. 110 8943-8953 (1999).

60. Frisch, M. J. et al. Gaussian 09, Revision A.02 (Gaussian, Inc., Wallingford CT, 2016).

61. Dennington, R., Keith, T. \& Millam, J. GaussView, Version 4.1 (Semichem, Inc., Shawnee Mission, 2007)
62. Aakeröy, C. B., Wijethunga, T. K., Desper, J. \& Đaković, M. Electrostatic potential differences and halogen-bond selectivity. Cryst. Growth Des. 16, 2662-2670 (2016)

\section{Acknowledgements}

P.M.J.S. and D.L.B. thank the Natural Sciences and Engineering Research Council of Canada for a scholarship and for research funding, respectively. The authors wish to thank Dr. Glenn A. Facey and Dr. Eric Ye for helpful discussions and assistance with the variable temperature experiments. The authors also thank Compute Canada for access to computing resources.

\section{Author contributions}

P.M.J.S. conceived and designed experiments, performed quantum chemical calculations, analyzed data, and prepared the manuscript. S.Z. prepared the samples, performed the variable temperature experiments, analyzed data, and revised the manuscript. D.L.B. conceived and designed experiments, supervised the work, provided guidance, and prepared the manuscript.

\section{Additional information}

Supplementary Information accompanies this paper at https://doi.org/10.1038/s41467019-08878-8.

Competing interests: The authors declare no competing interests.

Reprints and permission information is available online at http://npg.nature.com/ reprintsandpermissions/

Journal Peer Review Information: Nature Communications thanks Pierre Kennepohl and other anonymous reviewer(s) for their contribution to the peer review of this work.

Publisher's note: Springer Nature remains neutral with regard to jurisdictional claims in published maps and institutional affiliations.

(c) (i) Open Access This article is licensed under a Creative Commons By Attribution 4.0 International License, which permits use, sharing adaptation, distribution and reproduction in any medium or format, as long as you give appropriate credit to the original author(s) and the source, provide a link to the Creative Commons license, and indicate if changes were made. The images or other third party material in this article are included in the article's Creative Commons license, unless indicated otherwise in a credit line to the material. If material is not included in the article's Creative Commons license and your intended use is not permitted by statutory regulation or exceeds the permitted use, you will need to obtain permission directly from the copyright holder. To view a copy of this license, visit http://creativecommons.org/ licenses/by/4.0/.

(C) The Author(s) 2019 